\title{
Chemical chaperone 4-phenylbutyrate prevents endoplasmic reticulum stress induced by $\mathrm{T} 17 \mathrm{M}$ rhodopsin
}

\author{
Haibo Jiang, Siqi Xiong and Xiaobo Xia*
}

\begin{abstract}
Background: Rhodopsin mutations are associated with the autosomal dominant form of retinitis pigmentosa. T17M mutation in rhodopsin predisposes cells to endoplasmic reticulum (ER) stress and induces cell death. This study aimed to examine whether chemical chaperone 4-phenylbutyrate prevents ER stress induced by rhodopsin T17M.

Results: ARPE-19 cells were transfected with myc-tagged wild-type (WT) and T17M rhodopsin constructs. Turnover of WT and T17M rhodopsin was measured by cycloheximide chase analysis. The activity of ubiquitin-proteasome system was evaluated by GFPU reporter. We found that T17M rhodopsin was misfolded, ubiqutinated and eliminated by ER-associated degradation pathway (ERAD) in ARPE-19 cells. Accumulated T17M rhodopsin induced unfolded protein response, but had no effect on the activity of ubiquitin proteasome system. Moreover, chemical chaperone 4-phenylbutyrate facilitated the turnover of T17M rhodopsin and prevented apoptosis and ER stress induced by T17M rhodopsin.

Conclusions: Chemical chaperone could attenuate UPR signaling and ER stress induced by T17M rhodopsin and has potential therapeutic significance for retinitis pigmentosa.
\end{abstract}

Keyword: Retinitis pigmentosa, Rhodopsin, UPR, Misfolded, ERAD

\section{Background}

Retinitis pigmentosa (RP) is considered the most commonly inherited retinal dystrophy with an estimated prevalence of approximately 1:4000 [1]. RP is caused by the progressive loss of rod and cone photoreceptors with clinical hallmarks including the sensitivity to dim light, abnormal visual function and characteristic bone spicule deposits of pigment in the retina [2]. Mutations in rhodopsin, a photon receptor that initiates phototransduction, have been linked to autosomal dominant retinitis pigmentosa (ADRP), accounting for about $10 \%$ of all reported cases of RP $[3,4]$. Since the identification of the P23H mutation, more than 130 different mutations of rhodopsin have been shown to cause RP [5].

A mouse model of ADRP was created with a threonineto-methionine mutation at the 17 th residue of rhodopsin, which abolishes the glycosylation site at Asn15 and results

\footnotetext{
* Correspondence: xbxia21@163.com

Department of Ophthalmology, Xiangya Hospital, Central South University, 87 Xiangya Road, Changsha, Hunan 410078, P.R. China
}

in a class I RP phenotype [6,7]. Transgenic mice carrying human T17M rhodopsin gene showed significant photoreceptor apoptosis as early as $24 \mathrm{~h}$ after illumination, while mice expressing a rhodopsin transgene with $\mathrm{P} 23 \mathrm{H}$ mutation were only minimally affected [8]. Further study showed that endoplasmic reticulum (ER) stress response is involved in retinal degeneration in T17M rhodopsin retinas in vivo, accompanied by the up-regulation of autophagy markers and the activation of mitochondrial apoptosis via the up-regulation of pro-apoptotic $\mathrm{Bcl} 2$ [9]. Our previous study showed that T17M rhodopsin accumulated in ER, increased the cytotoxicity and predisposed the cells to ER stress induced cell death [10].

Misfolded proteins that do not pass ER quality control (ERQC) are selectively recognized and cleared during a process called ER-associated degradation (ERAD) that involves the export of misfolded proteins from ER followed by proteasomal degradation [11]. Up to now, the role of ERAD in the clearance of T17M rhodopsin is unclear. We proposed that T17M rhodopsin may be misfolded and eliminated via ERAD, and chemical chaperone 
4-phenylbutyrate may prevent ER stress induced by rhodopsin T17M.

In this study, we used a spontaneously arising retinal pigment epithelia (RPE) ARPE-19 cell line as the experimental model to investigate the role of ERAD in the clearance of $\mathrm{T} 17 \mathrm{M}$ rhodopsin and the effects of 4-phenylbutyrate (4-PBA) on ER stress induced by rhodopsin T17M.

\section{Methods}

\section{Cell culture and plasmid constructs}

WT and T17M rhodopsin-myc constructs were described previously [10]. p97/VCP QQ-HA construct was described previously [12]. ARPE-19 cells were obtained from ATCC and cultured with DMEM supplemented with 10\% FBS and penicillin-streptomycin $(50 \mu \mathrm{g} / \mathrm{ml})$ at $37^{\circ} \mathrm{C}$ in $5 \% \mathrm{CO}_{2}$. 4-PBA (Sigma, St. Louis, MO, USA) was dissolved in filtered sterile water at $1 \mathrm{M}$ stock concentration.

\section{Immunoblotting}

Cells were lysed with RIPA sample buffer, the supernatant was collected and protein concentration was determined using a Pierce protein assay kit (Thermo Scientific). $30 \mu \mathrm{g}$ proteins were separated on SDS-PAGE gel and transferred to PVDF membrane (Millipore). The membrane was incubated for $1 \mathrm{~h}$ in a blocking solution (5\% dry milk in $0.1 \%$ triton X-100/PBS buffer) followed by incubation with appropriate primary antibodies in a blocking solution. After being washed in $0.1 \%$ triton X-100/PBS buffer, the membrane was incubated in appropriate secondary antibodies for $1 \mathrm{~h}$ and visualized via an enhanced chemiluminescence kit (GE Health) according to the manufacturer's instruction. Antibodies were as follows: actin, HA antibodies (Abcam), Myc, Erasin, GRP78, GRP94, CHOP, peIF-2 $\alpha$, eIF- $2 \alpha$, and active ATF- $6 \alpha$ antibodies (Cell Signaling), GFP antibody (Invitrogen).

\section{Analysis of protein turnover}

ARPE-19 cells were transfected with erasin SMARTpool siRNAs (Dharmacon) using Dharmafect 1 reagent (Thermo Fisher Scientific) according to the manufacturer's instructions. $48 \mathrm{~h}$ later, cells were transfected with Myc-tagged rhpdopsin using lipofection 2000 (Invitrogen). After $24 \mathrm{~h}$, cells were treated with $50 \mu \mathrm{g} / \mathrm{ml}$ cycloheximide (Sigma)

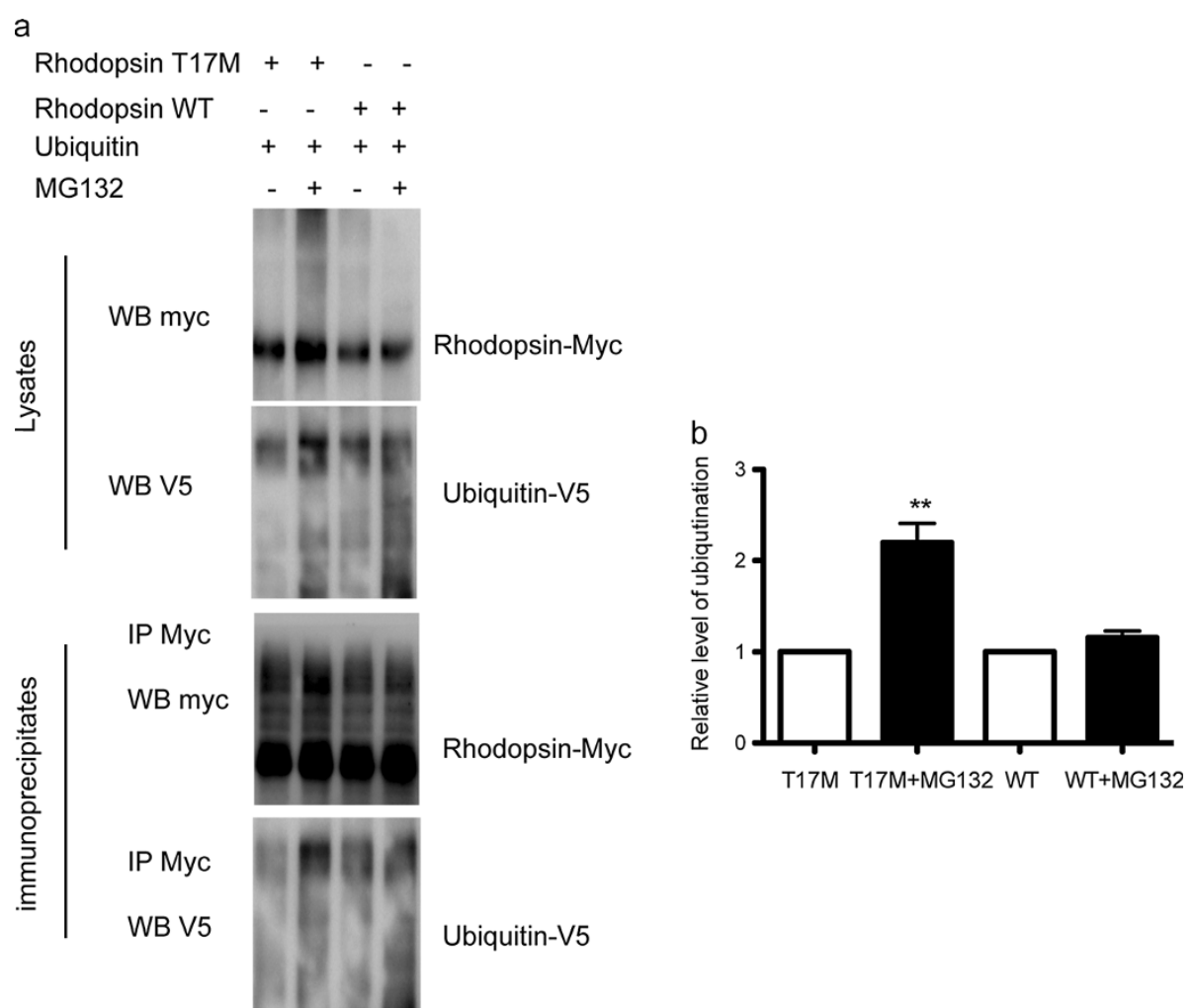

Figure 1 Ubiquitination of myc-tagged rhodopsin T17M. (a). Upper: ARPE-19 cells were transfected with myc-tagged rhodopsin T17M or WT expression constructs. At $24 \mathrm{~h}$ post-transfection, the cells were treated with MG132 (10 $\mu \mathrm{M})$ or DMSO for $6 \mathrm{~h}$. The protein was detected by immunoblotting. Lower: Immunoprecipitation of transfected myc-tagged rhodopsin T17M or WT from ARPE-19 cells after MG132 treatment. The immunoprecipitates were immunoblotted for myc and ubiquitin-V5 (b). Quantification of the relative level of ubiquitination. Data were presented as mean \pm S.D. ${ }^{* *} p<0.01$ : compared to T17M cells. 
and collected at the indicated time points. For turnover experiments with $\mathrm{p} 97 / \mathrm{VCP}$ QQ-HA, DNA construct was co-transfected with Myc-tagged rhpdopsin plasmid DNA followed by the inhibition of protein synthesis with cycloheximide. Equal amounts of protein in lysates from the different time points were separated by SDS-PAGE and immunoblotted.

\section{RNA extraction, RT-PCR and XBP1 splicing assay}

Total RNA was extracted from cells with TRIzol (Invitrogen) and cDNA was synthesized with a High Capacity cDNA Reverse Transcription kit (Applied Biosystems). Primers encompassing the spliced sequences in XBP1 mRNA (5'-ACACGCTTGGGAATGGACAC-3' and 5'CCATGGGAAGATGTTCTGGG-3') and loading control Actin (5'-GCGAGAAGATGACCCAGATC-3', and 5'-CCAGTGGTACGGCCAGAGG-3') were used for PCR, and products were separated by electrophoresis through a $2.5 \%$ agarose gel and visualized by ethidium bromide staining.

\section{Apoptosis detection}

ARPE-19 cells were transfected with myc-tagged wild-type or T17M mutant constructs. Twenty hours after transfection, apoptosis was detected by Annexin V-FITC Apoptosis Detection Kit (Sigma). Briefly, cells were collected and washed twice with PBS. Then cells were resuspended in $500 \mu \mathrm{L}$ Binding Buffer, and incubated with the addition of $5 \mu \mathrm{L}$ Annex V-FITC and $10 \mu \mathrm{L}$ propidium iodide at $37^{\circ} \mathrm{C}$ for $15 \mathrm{~min}$ in the dark. Next the stained samples were subjected to flow cytometry analysis to detect the apoptosis rate.

\section{Determination of cellular $26 \mathrm{~S}$ proteasome activity}

A stable cell line overexpressing $\mathrm{GFP}^{\mathrm{U}}$ in ARPE-19 cells was established as a cellular model to test $26 \mathrm{~S}$ proteasome activity after G418 selection. The reporter gene consisted of a short tag CL1 fused to the C-terminus of GFP. CL1, encoding a fragment of amino acids (ACKNWFSSLSHFVIHL), was shown to be a degradation substrate for ubiquitin-proteasome system [13]. The expression level of

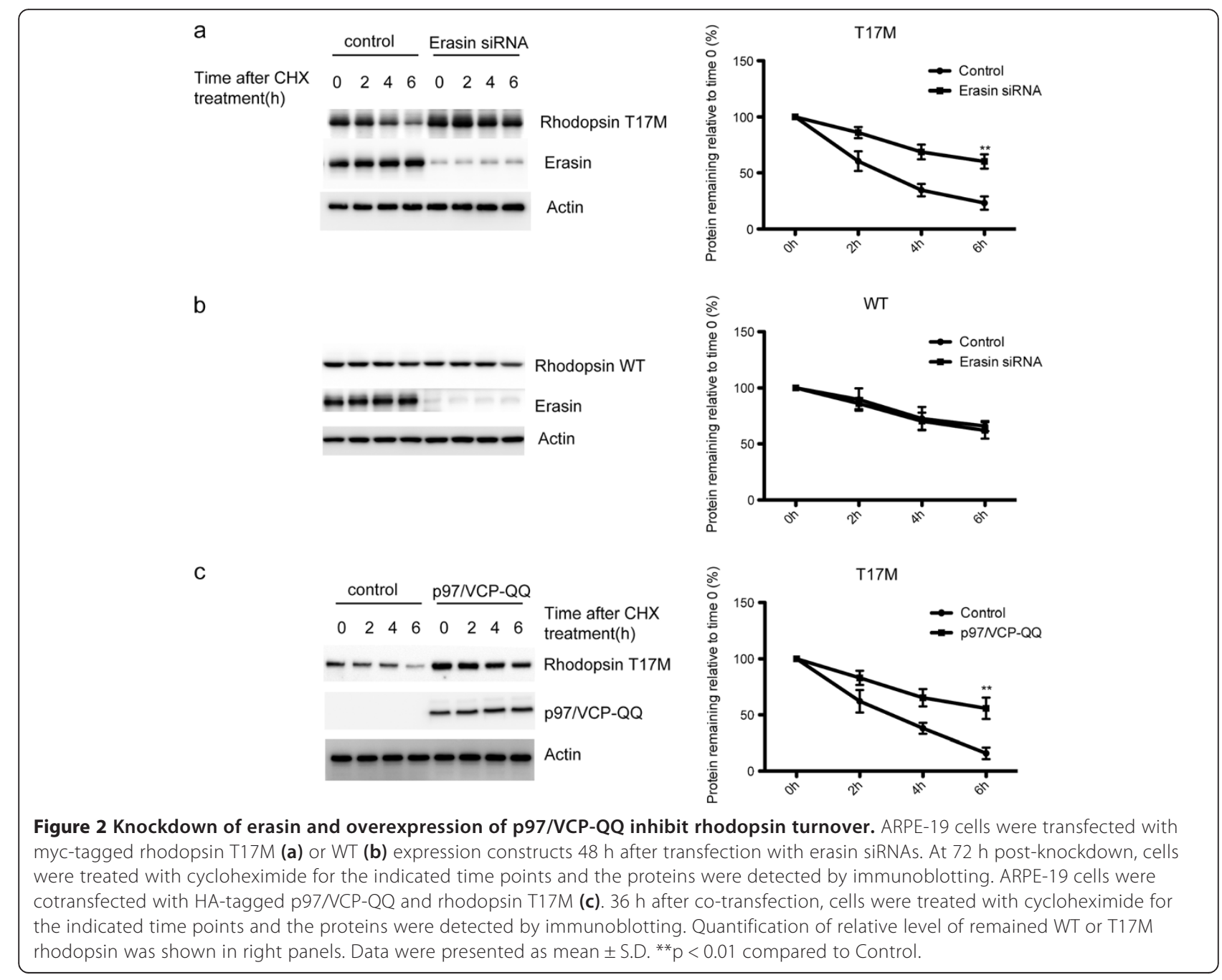


$\mathrm{GFP}^{\mathrm{U}}$ after the transfection of rhodopsin $\mathrm{T} 17 \mathrm{M}$ or WT was determined by immunoblotting.

\section{Statistical analysis}

All experiments were repeated at least three times. Results were presented as means \pm standard deviation (SD). Statistical significance of differences was evaluated with one way ANOVA followed by the Tukey test and Dunnett's test. $\mathrm{p}<0.05$ was considered as statistical significance. GraphPad Prism 5 software was used for the analysis.

\section{Results}

\section{Rhodopsin T17M mutant protein is ubiquitinated}

Proteins that are terminally misfolded in the ER may be degraded by the proteasome via ERAD. Typically, such proteins are unstable and show a reduced half-life. Because ERAD substrates are typically ubiquitinated [14], we examined whether rhodopsin T17M protein is modified by ubiquitination. We immunoprecipitated Myc-tagged wild-type and T17M mutant rhodopsin proteins from ARPE-19 cells using myc antibody and probed the immunoprecipitates. As shown in Figure 1, considerably more rhodopsin $\mathrm{T} 17 \mathrm{M}$ protein was observed in cell treated with MG132 than in untreated cells, confirming that proteasome inhibition leads to the stabilization of rhodopsin T17M. After immunoprecipitation and normalization, we observed a smear on the upper portions of the gels by using ubiquitin antibody, especially after MG312 treatment, indicating that rhodopsin T17M protein is modified by ubiquitination.

\section{Rhodopsin T17M mutant protein is degraded via ERAD pathway}

To examine whether rhodopsin T17M mutant protein is eliminated by ERAD pathway, we disrupted two components involved in ERAD pathway to see how this would affect the turnover of rhodopsin T17M protein. The first protein we targeted was erasin. Erasin promoted ERAD. Overexpression of erasin enhanced the degradation of ERAD substrates, whereas siRNA-mediated reduction of erasin expression almost completely blocked ERAD [15]. We transfected ARPE-19 cells with myc-tagged WT or rhodopsin T17M after siRNA-mediated knockdown of erasin, and assessed protein turnover by cycloheximide chase analysis. The results showed that rhodopsin T17M was readily degraded with a half-life of around $4 \mathrm{~h}$, whereas it was stable over the $6 \mathrm{~h}$ chase period in erasin knockdown cells. Disruption of erasin also induced increased rhodopsin $\mathrm{T} 17 \mathrm{M}$ at $0 \mathrm{~h}$ cycloheximide treatment (Figure 2a). In contrast, knockdown of erasin had no significant effect on the turnover of rhodopsin WT protein (Figure 2b).

We next examined p97/VCP, which is essential for the dislocation of proteins from ER during ERAD [16]. We utilized a dominant-negative ATPase deficient p97/VCPQQ mutant, which has been shown to slow the degradation of ERAD substrates. Accordingly, we cotransfected ARPE19 cells with HA-tagged p97/VCP-QQ and myc-tagged WT or rhodopsin T17M, and assessed protein turnover by cycloheximide chase analysis. The results showed that the amounts of rhodopsin T17M was much higher in p97/VCP-QQ overexpressing cells than in control cells, indicating that $\mathrm{p} 97 / \mathrm{VCP}-\mathrm{QQ}$ inhibited the turnover of rhodopsin T17M (Figure 2c). Taken together, these results strongly indicate that rhodopsin T17M mutant protein is degraded via ERAD pathway.

\section{Rhodopsin T17M mutant protein has no effect on ubiquitin-proteasome system}

Ubiquitin-proteasome system (UPS) dysfunction is an important pathogenic factor in neurodegeneration diseases. The most common rhodopsin mutation $\mathrm{P} 23 \mathrm{H}$ forms aggregates in ER and impairs UPS [17]. To investigate the effect of rhodopsin T17M on proteasome, ARPE-19 cells with stable expression of a GFP-conjugated proteasome degradation signal $\mathrm{GFP}^{\mathrm{U}}$ was used [13]. The $\mathrm{GFP}^{\mathrm{U}}$ reporter consists of a short degron, CL1, fused to the Cterminus of GFP. The product of $\mathrm{GFP}^{\mathrm{U}}$ was continuously

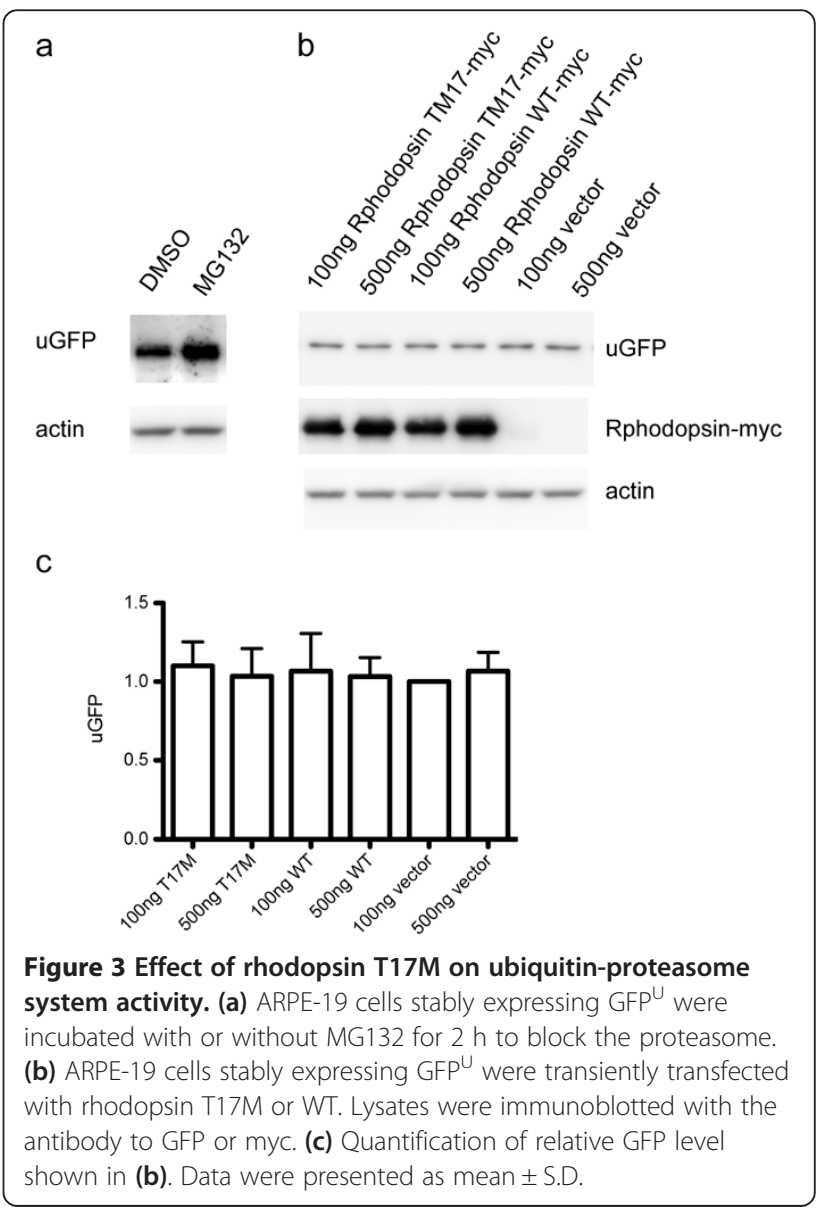


degraded and kept at a very low level under normal conditions. A decline of $\mathrm{GFP}^{\mathrm{U}}$ level reflects ubiquitinproteasome system activity, whereas an increase of level indicates that UPS activity is reduced or impaired. We have established a $\mathrm{GFP}^{\mathrm{U}}$ stable expression ARPE-19 cell line using G418 selection. The GFP ${ }^{\mathrm{U}}$ protein was significantly increased when proteasome was blocked by MG132, a proteasome inhibitor (Figure 3a). Then, ARPE19 cells were transiently transfected with WT or rhodopsin $\mathrm{T} 17 \mathrm{M}$, and $\mathrm{GFP}^{\mathrm{U}}$ level was detected. The results showed that there were no significant differences in GFPU level between WT and rhpdopsin T17M (Figure 3), indicating that Rhodopsin T17M mutant protein has no effect on UPS activity.

\section{Rhodopsin T17M mutant protein induces unfolded protein response}

The accumulation of misfolded proteins in ER can activate unfolded protein response (UPR). To determine whether misfolding of rhodopsin T17M would activate UPR, ARPE-19 cells were transiently transfected with WT or rhodopsin T17M expression vector, and the levels of ER chaperone and UPR-associated proteins such as GRP78, GRP94, CHOP, peIF- $2 \alpha$, eIF- $2 \alpha$, active ATF-6 $\alpha$ were assessed by immunoblotting. Compared to control, rhodopsin T17M mutant increased the levels of these proteins (Figure 4).

\section{4-phenylbutyrate prevents UPR induced by rhodopsin T17M}

4-phenylbutyrate (4-PBA) is a small chemical chaperone that could reduce ER stress both in vivo and in vitro. Thus we used PBA to treat ARPE-19 cells overexpressing rhodopsin T17M. Western blot analysis showed that the levels of GRP78, GRP94, CHOP, peIF- $2 \alpha$, eIF- $2 \alpha$, and active ATF- $6 \alpha$ were reduced after 4-PBA treatment (Figure $4 \mathrm{a}, \mathrm{b}$ ). We also detected the splicing of XBP-1 in ARPE-19 cells overexpressing rhodopsin T17M. The small size form of XBP-1(XBP-1 s) was observed in rhodopsin T17M overexpressing cells. As expected, 4-PBA reduces the splicing of XPB-1 induced by rhodopsin T17M (Figure 4c, d). Taken together, these data suggested that 4-PBA attenuates UPR signaling and ER stress induced by rhodopsin T17M in ARPE-19 cells.

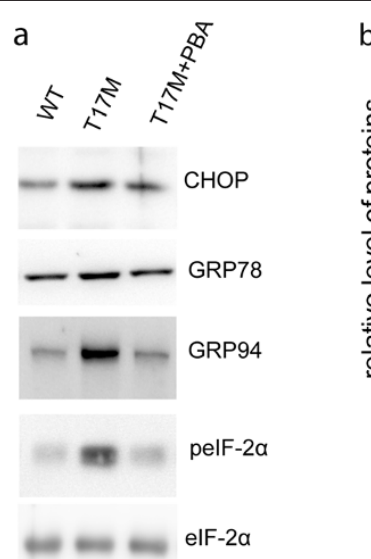

b

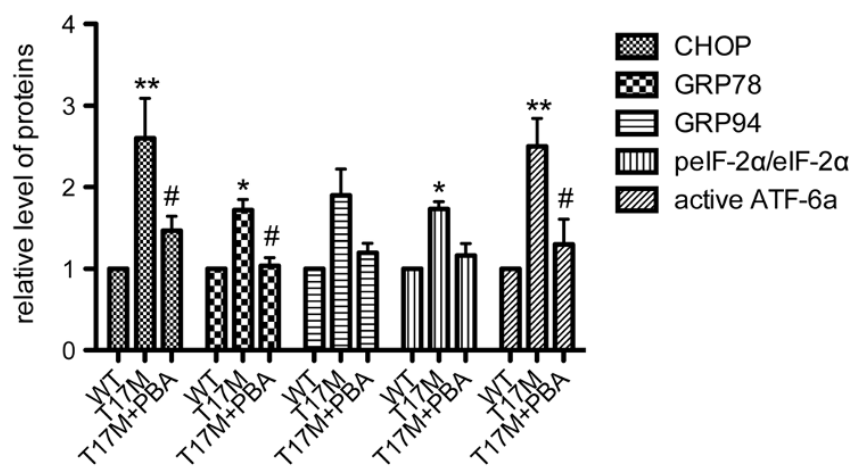

d

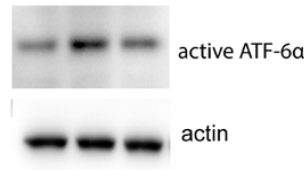

C

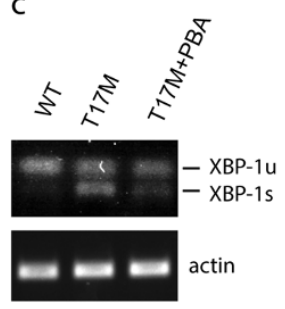

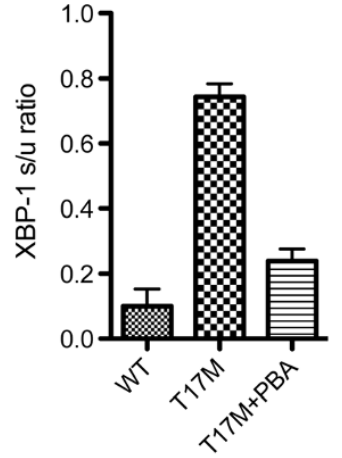

Figure 4 4-PBA alleviates UPR and ER stress induced by rhodopsin T17M mutant. ARPE-19 cells were transfected with myc-tagged rhodopsin T17M or WT expression constructs and treated with $5 \mathrm{mM} 4$-PBA. After $24 \mathrm{~h}$, the indicated proteins (a) and splicing of XBP-1(c) were detected by immunoblotting and RT-PCR, respectively. Quantification of the data in (a) and (b) were shown in (c) and (d), respectively. Data were presented as mean \pm S.D. *:T17M vs. Control; \#: T17M + 4-PBA vs. T17M; ${ }^{* *} p<0.01$.; ${ }^{*} p<0.05 ; \# p<0.05$. 


\section{Phenylbutyric acid facilitates rhodopsin T17M degradation and inhibits apoptosis induced by rhodopsin T17M}

To investigate whether 4-PBA has an effect on the turnover of rhodopsin T17M protein, we performed cycloheximide chase analysis. Upon 4-PBA treatment rhodopsin T17M had a short half-life of $\sim 3 \mathrm{~h}$, compared to $\sim 4 \mathrm{~h}$ half-life in absence of 4-PBA (Figure 5a, b). However, we found that 4-PBA had no significant effect on intracellular localization of rhodopsin T17M in ARPE-19 cells (data not shown). Rhodopsin T17M protein is known to accumulate in ER and induce ER stress. Thus we wondered whether 4-PBA had protective effect on apoptosis induced by rhodopsin T17M. The results showed that the apoptosis rate was significantly higher in cells transfected with rhodopsin T17M expression vector than in cells transfected with empty vector. However, 4-PBA partially inhibited apoptosis induced by the overexpression of rhodopsin T17M (Figure 5c, d).

\section{Discussion}

In this study, we found that rhodopsin T17M protein was ubiquitinated and the ubiquitination was increased following proteasome inhibitor treatment. Moreover, interference of ERAD either by overexpression of a dominant negative $\mathrm{p} 97 / \mathrm{VCP}-\mathrm{QQ}$ protein or by knockdown of erasin slowed the degradation of rhodopsin T17M mutant protein.
RPE is a monolayer of hexagonal cells separating the neural retina from the underlying choroidal vascular bed. RPE cells are essential for the development, survival, and physiological activity of photoreceptor cells [18]. Mutations in genes that are expressed in the RPE can lead to photoreceptor degeneration. On the other hand, mutations in genes expressed in photoreceptor cells can lead to degenerations of the RPE. Thus RPE and photoreceptors cells are closely linked [19]. It is found that rhodopsin is expressed in RPE cells [20]. The human retinal pigment epithelial cell line (ARPE-19), a transformed human retinal pigment epithelial cell line, was employed to investigate the effect of rhodopsin mutant on RPE degeneration. In addition, some mammalian cell lines, such as 293 s [21], HeLa [22] and COS [23] were used to investigate biological functions of rhodopsin involved in RP mechanism.

T17M is a type II mutant rhodopsin that traffics abnormally and forms pigment inefficiently [24]. Proper protein folding and processing is necessary to maintain cellular homeostasis. Protein misfolding could potentially not only affect function but also lead to protein aggregation and induce toxicity. Not surprisingly, cells have developed elaborate and complex systems to eliminate unwanted and potentially toxic proteins. One of the first quality control checkpoints is in the ER, where misfolded proteins are recognized and eliminated by ERAD. Although

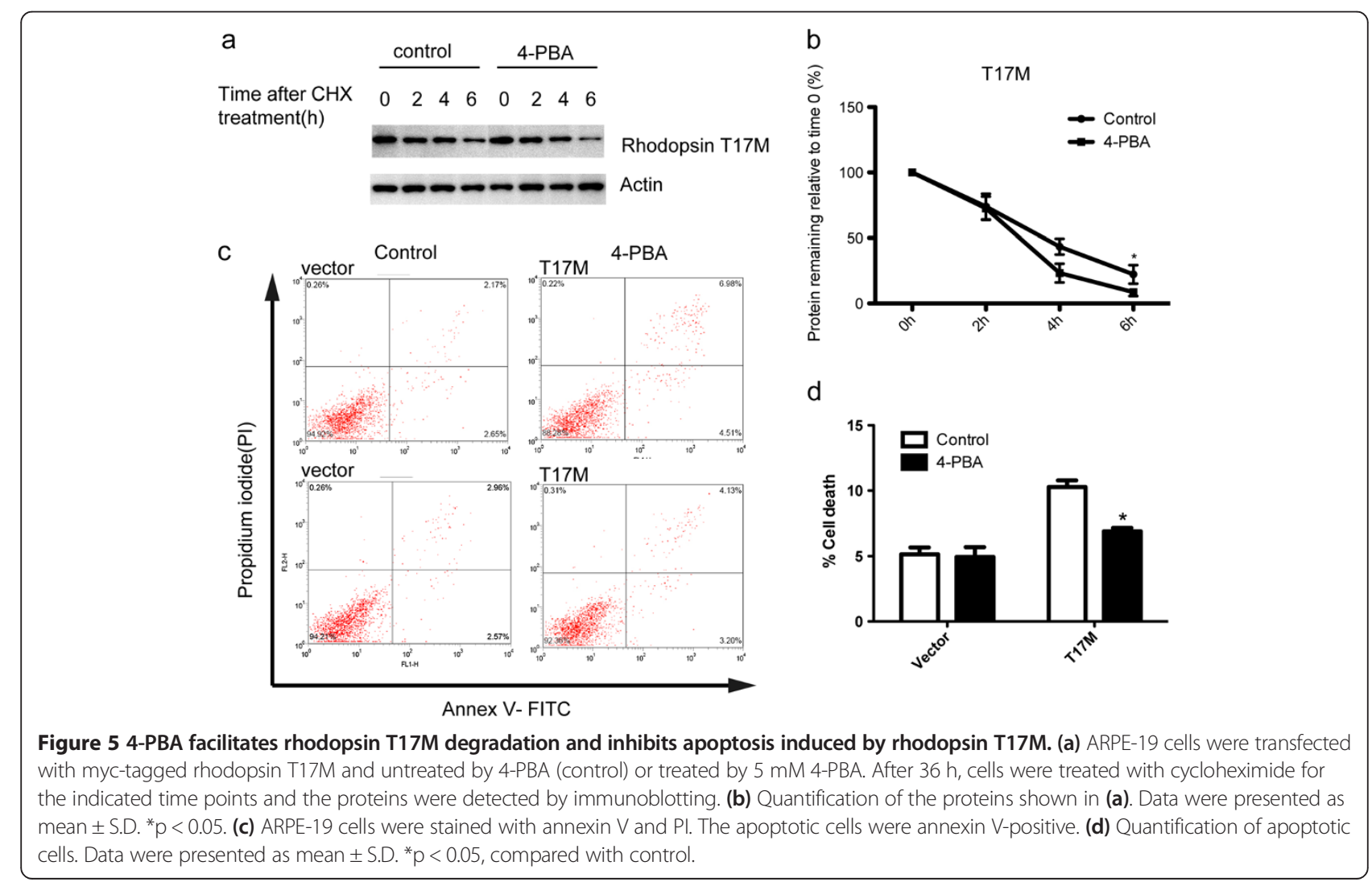


many mutations involved in human disease are thought to cause proteins to misfold, relatively few of them have been shown to be eliminated by ERAD. Here, we presented evidence that mutant rhodopsin protein linked to RP is degraded by ERAD.

Misfolded rhodopsin $\mathrm{R} 32 \mathrm{H}$ is a substrate of the ERAD effector VCP, an ATP-dependent chaperone that extracts misfolded proteins from the ER and escorts them for proteasomal degradation [25]. Inactivation of $\mathrm{VCP} /$ ter94 suppresses retinal pathology caused by misfolded rhodopsin in Drosophila [26]. Co-expression of certain ERAD factors was sufficient to reduce Rh-1 protein levels and suppress ER stress reporter activation, indicating that ERAD acts as a protective mechanism against retinal degeneration in the Drosophila model for ADRP. These results suggest that manipulation of ERAD may serve as a powerful therapeutic strategy against a number of diseases associated with ER stress [27].

ER is responsible for the folding of secreted and membrane proteins in eukaryotic cells. Disruption of protein folding leads to ER stress. Chronic ER stress can cause cell death and is implicated in the pathogenesis of many human diseases. ER stress response is involved in retinal degeneration in T17M Rho mice and S334ter Rho rats [28]. Ablation of CHOP, selective activation of ATF6 or PERK, disruption of CDK5 and MEKK1 pathway, or the stimulation of ERAD may serve as a powerful therapeutic strategy against rhodopsin mutants induced RP and reverse severe retinal degeneration [29-32]. Chemical chaperones reduce ER stress and are proposed as therapeutic target for various diseases [33]. 4-PBA is a low molecular weight terminal aromatic substituted fatty acid approved for clinical use as an ammonia scavenger in children with urea cycle disorders [34]. Recent reports showed that 4-PBA has protective effects against ER stress-induced neuronal cell death $[35,36]$. In this study our results showed that 4-PBA reduced ER stress induced by T17M rhodopsin. Thus 4-PBA is a promising agent to treat RP because it could prevent RP induced by T17M rhodopsin mutant. Although 4-PBA facilitates the degradation of $\mathrm{T} 17 \mathrm{M}$ mutant and attenuates apoptosis induced by ER stress, it does not affect the location of T17M mutant. Further studies are necessary to investigate whether intracellular trafficking of immature or misfolded rhodopsin is a potential target for RP therapy.

\section{Conclusions}

T17M rhodopsin is misfolded, ubiquitinated and eliminated by ER-associated degradation pathway. Chemical chaperone could attenuate UPR signaling and ER stress induced by T17M rhodopsin and has potential therapeutic significance for retinitis pigmentosa.
Competing interests

The authors declare that they have no competing interests.

\section{Authors' contributions}

$\mathrm{HJ}$ and XX conceived the study, HJ and SX performed the experiments and drafted the manuscript. All authors read and approved the final manuscript.

\section{Acknowledgements}

This study was supported by the grant from the National Natural Science Foundation of China (No. 81170844) and Hunan Provincial Science \& Technology Department (No. 2014FJ3100).

Received: 5 September 2014 Accepted: 25 November 2014

Published: 4 December 2014

\section{References}

1. Rosenberg T: Epidemiology of hereditary ocular disorders. Dev Ophthalmol 2003, 37:16-33.

2. Dryja TP, McGee TL, Hahn LB, Cowley GS, Olsson JE, Reichel E, Sandberg MA, Berson EL: Mutations within the rhodopsin gene in patients with autosomal dominant retinitis pigmentosa. N Engl J Med 1990, 323:1302-1307.

3. Hartong DT, Berson EL, Dryja TP: Retinitis pigmentosa. Lancet 2006, 368:1795-1809.

4. Rivolta C, Sharon D, DeAngelis MM, Dryja TP: Retinitis pigmentosa and allied diseases: numerous diseases, genes, and inheritance patterns. Hum Mol Genet 2002, 11:1219-1227.

5. Berson EL: Retinitis pigmentosa: unfolding its mystery. Proc Natl Acad Sci U S A 1996, 93:4526-4528.

6. Li T, Sandberg MA, Pawlyk BS, Rosner B, Hayes KC, Dryja TP, Berson EL: Effect of vitamin A supplementation on rhodopsin mutants threonine-17 methionine and proline-347 serine in transgenic mice and in cell cultures. Proc Natl Acad Sci U S A 1998, 95:11933-11938.

7. Kaushal S, Khorana HG: Structure and function in rhodopsin. 7. Point mutations associated with autosomal dominant retinitis pigmentosa. Biochemistry 1994, 33:6121-6128.

8. White DA, Fritz JJ, Hauswirth WW, Kaushal S, Lewin AS: Increased sensitivity to light-induced damage in a mouse model of autosomal dominant retinal disease. Invest Ophthalmol Vis Sci 2007, 48:1942-1951.

9. Kunte MM, Choudhury S, Manheim JF, Shinde VM, Miura M, Chiodo VA, Hauswirth WW, Gorbatyuk OS, Gorbatyuk MS: ER stress is involved in T17M rhodopsin-induced retinal degeneration. Invest Ophthalmol Vis Sci 2012, 53:3792-3800

10. Jiang $H$, Xiong $S$, Xia $X$ : Retinitis pigmentosaassociated rhodopsin mutant T17M induces endoplasmic reticulum (ER) stress and sensitizes cells to ER stress-induced cell death. Mol Med Rep 2014, 9:1737-1742.

11. Romisch K: Endoplasmic reticulum-associated degradation. Annu Rev Cell Dev Biol 2005, 21:435-456.

12. Zhong $X$, Shen $Y$, Ballar $P$, Apostolou A, Agami R, Fang S: AAA ATPase p97/valosin-containing protein interacts with gp78, a ubiquitin ligase for endoplasmic reticulum-associated degradation. J Biol Chem 2004, 279:45676-45684.

13. Gilon T, Chomsky O, Kulka RG: Degradation signals for ubiquitin system proteolysis in Saccharomyces cerevisiae. EMBO J 1998, 17:2759-2766.

14. Hampton RY: ER-associated degradation in protein quality control and cellular regulation. Curr Opin Cell Biol 2002, 14:476-482.

15. Liang J, Yin C, Doong H, Fang S, Peterhoff C, Nixon RA, Monteiro MJ: Characterization of erasin (UBXD2): a new ER protein that promotes ER-associated protein degradation. J Cell Sci 2006, 119:4011-4024.

16. DeLaBarre B, Christianson JC, Kopito RR, Brunger AT: Central pore residues mediate the p97NCP activity required for ERAD. Mol Cell 2006, 22:451-462.

17. Griciuc A, Aron L, Piccoli G, Ueffing M: Clearance of Rhodopsin(P23H) aggregates requires the ERAD effector VCP. Biochim Biophys Acta 1803, 2010:424-434.

18. Sharma RK, Johnson DA: Molecular signals for development of neuronal circuitry in the retina. Neurochem Res 2000, 25:1257-1263.

19. Strauss O: The retinal pigment epithelium in visual function. Physio/ Rev 2005, 85:845-881.

20. Strunnikova NV, Maminishkis A, Barb JJ, Wang F, Zhi C, Sergeev $Y$, Chen W, Edwards AO, Stambolian D, Abecasis G, Swaroop A, Munson PJ, Miller SS: Transcriptome analysis and molecular signature of human retinal pigment epithelium. Hum Mol Genet 2010, 19:2468-2486. 
21. Opefi CA, South K, Reynolds CA, Smith SO, Reeves PJ: Retinitis pigmentosa mutants provide insight into the role of the N-terminal cap in rhodopsin folding, structure, and function. I Biol Chem 2013, 288:33912-33926.

22. Yamasaki A, Hara T, Maejima I, Sato M, Sato K, Sato K: Rer1p regulates the ER retention of immature rhodopsin and modulates its intracellular trafficking. Sci Rep 2014, 4:5973.

23. Zhao Y, Hong DH, Pawlyk B, Yue G, Adamian M, Grynberg M, Godzik A, Li T: The retinitis pigmentosa GTPase regulator (RPGR)- interacting protein: subserving RPGR function and participating in disk morphogenesis. Proc Natl Acad Sci U S A 2003, 100:3965-3970.

24. Krebs MP, Holden DC, Joshi P, Clark CL 3rd, Lee AH, Kaushal S: Molecular mechanisms of rhodopsin retinitis pigmentosa and the efficacy of pharmacological rescue. J Mol Biol 2010, 395:1063-1078.

25. Kroeger $\mathrm{H}$, Chiang WC, Lin JH: Endoplasmic reticulum-associated degradation (ERAD) of misfolded glycoproteins and mutant P23H rhodopsin in photoreceptor cells. Adv Exp Med Biol 2012, 723:559-565.

26. Griciuc A, Aron L, Roux MJ, Klein R, Giangrande A, Ueffing M: Inactivation of VCP/ter94 suppresses retinal pathology caused by misfolded rhodopsin in Drosophila. PLoS Genet 2010, 6(8). doi:10.1371/journal.pgen.1001075.

27. Kang MJ, Ryoo HD: Suppression of retinal degeneration in Drosophila by stimulation of ER-associated degradation. Proc Natl Acad Sci U S A 2009, 106:17043-17048.

28. Shinde VM, Sizova OS, Lin JH, LaVail MM, Gorbatyuk MS: ER stress in retinal degeneration in S334ter Rho rats. PLoS One 2012, 7:e33266.

29. Chiang WC, Hiramatsu N, Messah C, Kroeger H, Lin JH: Selective activation of ATF6 and PERK endoplasmic reticulum stress signaling pathways prevent mutant rhodopsin accumulation. Invest Ophthalmol Vis Sci 2012, 53:7159-7166

30. Kang MJ, Chung J, Ryoo HD: CDK5 and MEKK1 mediate pro-apoptotic signalling following endoplasmic reticulum stress in an autosomal dominant retinitis pigmentosa model. Nat Cell Biol 2012, 14:409-415.

31. Chiang WC, Messah C, Lin JH: IRE1 directs proteasomal and lysosomal degradation of misfolded rhodopsin. Mol Biol Cell 2012, 23:758-770.

32. Choudhury S, Bhootada Y, Gorbatyuk O, Gorbatyuk M: Caspase-7 ablation modulates UPR, reprograms TRAF2-JNK apoptosis and protects T17M rhodopsin mice from severe retinal degeneration. Cell Death Dis 2013, 4:e528.

33. Minamino T, Komuro I, Kitakaze M: Endoplasmic reticulum stress as a therapeutic target in cardiovascular disease. Circ Res 2010, 107:1071-1082.

34. Brusilow SW, Maestri NE: Urea cycle disorders: diagnosis, pathophysiology, and therapy. Adv Pediatr 1996, 43:127-170.

35. Kubota K, Niinuma Y, Kaneko M, Okuma Y, Sugai M, Omura T, Uesugi M, Uehara T, Hosoi T, Nomura Y: Suppressive effects of 4-phenylbutyrate on the aggregation of Pael receptors and endoplasmic reticulum stress. J Neurochem 2006, 97:1259-1268.

36. Mimori S, Okuma Y, Kaneko M, Kawada K, Hosoi T, Ozawa K, Nomura Y, Hamana H: Protective effects of 4-phenylbutyrate derivatives on the neuronal cell death and endoplasmic reticulum stress. Biol Pharm Bull 2012, 35:84-90.

doi:10.1186/2045-3701-4-75

Cite this article as: Jiang et al:: Chemical chaperone 4-phenylbutyrate prevents endoplasmic reticulum stress induced by T17M rhodopsin. Cell \& Bioscience 2014 4:75.

\section{Submit your next manuscript to BioMed Central and take full advantage of:}

- Convenient online submission

- Thorough peer review

- No space constraints or color figure charges

- Immediate publication on acceptance

- Inclusion in PubMed, CAS, Scopus and Google Scholar

- Research which is freely available for redistribution 